\title{
Evidence of episodic positive selection in Corynebacterium diphtheriae complex of species and its implementations in identification of drug and vaccine targets
}

\author{
Marcus Vinicius Canário Viana ${ }^{1,2}$, Rodrigo Profeta ${ }^{1}$, Janaína C Cerqueira ${ }^{1}$, Alice Rebecca Wattam ${ }^{3}$, Debmalya \\ Barh $^{1,4}$, Artur Silva ${ }^{2}$, Vasco Azevedo ${ }^{\text {Corresp. } 1}$ \\ ${ }^{1}$ Departamento de Genética, Ecologia e Evolução, Universidade Federal de Minas Gerais, Belo Horizonte, Minas Gerais, Brazil \\ 2 Departamento de Genética, Universidade Federal do Pará, Belém, Pará, Brazil \\ 3 Biocomplexity Institute, University of Virginia, Charlottesville, Virginia, United States \\ 4 Institute of Integrative Omics and Applied Biotechnology, Nonakuri, West Bengal, India \\ Corresponding Author: Vasco Azevedo \\ Email address: vasco@icb.ufmg.br
}

Background. Within the pathogenic bacterial species Corynebacterium genus, six species that can produce diphtheria toxin (C. belfantii, C. diphtheriae, C. pseudotuberculosis, $C$. rouxii, $C$. silvaticum and $C$. ulcerans) form a clade referred to as the $C$. diphtheria complex. These species have been found in humans and other animals, causing diphtheria or other diseases. Here we show the results of a genome scale analysis to identify positive selection in protein-coding genes that may have resulted in the adaptations of these species to their ecological niches and suggest drug and vaccine targets. Methods. Forty genomes were sampled to represent species, subspecies or biovars of Corynebacterium. Ten phylogenetic groups were tested for positive selection using the PosiGene pipeline, including species and biovars from the $C$. diphtheria complex. The detected genes were tested for recombination and had their sequences alignments and homology manually examined. The final genes were investigated for their function and a probable role as vaccine or drug targets. Results. Nineteen genes were detected in the species $C$. diphtheriae (2), C. pseudotuberculosis (10), C. rouxii (1), and C. ulcerans (6). Those were found to be involved in defense, translation, energy production, and transport and in the metabolism of carbohydrates, amino acids, nucleotides, and coenzymes. Fourteen were identified as essential genes, and six as virulence factors. Thirteen from the 19 genes were identified as potential drug targets and 4 as potential vaccine candidates. These genes could be important in the prevention and treatment of the diseases caused by these bacteria. 
1 Evidence of episodic positive selection in Corynebacterium

2 diphtheriae complex of species and its implementations in

3 identification of drug and vaccine targets

4

5 Marcus Vinicius Canário Viana ${ }^{1,2}$, Rodrigo Profeta ${ }^{1}$, Janaína Canário Cerqueira ${ }^{1}$, Alice Rebecca

6 Wattam $^{3}$, Debmalya Barh ${ }^{1,4}$, Artur Silva², Vasco Azevedo ${ }^{1, *}$

7

9

${ }^{1}$ Departamento de Genética, Ecologia e Evolução, Instituto de Ciências Biológicas, Universidade Federal de Minas Gerais, 31270-901, Belo Horizonte, Minas Gerais, Brazil

${ }^{2}$ Departamento de Genética, Instituto de Ciências Biológicas, Universidade Federal do Pará, Belém, Pará, Brazil

${ }^{3}$ Biocomplexity Institute, University of Virginia, Charlottesville, Virginia, USA

${ }^{4}$ Institute of Integrative Omics and Applied Biotechnology, Nonakuri, Purba Medinipur, West Bengal, India

\section{Corresponding Author:}

Vasco Azevedo ${ }^{1}$

Av. Presidente Antônio Carlos 6627, Belo Horizonte, Minas Gerais, 31270-901, Brazil

Email address: vasco@icb.ufmg.br

\section{Abstract}

Background. Within the pathogenic bacterial species Corynebacterium genus, six species that can produce diphtheria toxin (C. belfantii, C. diphtheriae, C. pseudotuberculosis, C. rouxii, C. silvaticum and $C$. ulcerans) form a clade referred to as the $C$. diphtheria complex. These species have been found in humans and other animals, causing diphtheria or other diseases. Here we show the results of a genome scale analysis to identify positive selection in protein-coding genes that may have resulted in the adaptations of these species to their ecological niches and suggest drug and vaccine targets.

Methods. Forty genomes were sampled to represent species, subspecies or biovars of Corynebacterium. Ten phylogenetic groups were tested for positive selection using the PosiGene pipeline, including species and biovars from the $C$. diphtheria complex. The detected genes were tested for recombination and had their sequences alignments and homology manually examined. The final genes were investigated for their function and a probable role as vaccine or drug targets.

Results. Nineteen genes were detected in the species C. diphtheriae (2), C. pseudotuberculosis (10), C. rouxii (1), and C. ulcerans (6). Those were found to be involved in defense, translation, energy production, and transport and in the metabolism of carbohydrates, amino acids, nucleotides, and coenzymes. Fourteen were identified as essential genes, and six as virulence factors. Thirteen from the 19 genes were identified as potential drug targets and 4 as potential 
39

40

41

42

43

44

45

46

47

48

49

50

51

52

53

54

55

56

57

58

59

60

61

62

63

64

65

66

67

68

69

70

71

72

73

74

75

76

77

78

79

80

81

82

83

vaccine candidates. These genes could be important in the prevention and treatment of the diseases caused by these bacteria.

\section{Introduction}

The genus Corynebacterium are gram-positive bacteria of biotechnological, medical, and veterinary importance (Bernard \& Funke, 2015). Within the pathogenic species, some can produce diphtheria toxin (DT) after lysogenization by tox+ corynephages (Bernard \& Funke, 2015). Three species that compose a clade were initially described as potential diphtheria toxin (DT) producers: C. diphtheriae, C. ulcerans and C. pseudotuberculosis (Bernard \& Funke, 2015). The number of species in the clade of potential DT producers increased to six with the inclusion of the recently described C. belfantii (Dazas et al., 2018), C. rouxii (Badell et al., 2020) and C. silvaticum (Dangel et al., 2020). Those six species are described here as the " $C$. diphtheria complex" (Badell et al., 2020).

C. diphtheriae, C. belfantii and C. rouxii infect mainly humans (Bernard \& Funke, 2015; Dazas et al., 2018; Badell et al., 2020). C. ulcerans, C. pseudotuberculosis and C. silvaticum infect mainly wild and domesticated mammals and/or can cause zoonosis (Bernard \& Funke, 2015; Dangel et al., 2020). C. belfantii and C. rouxii have recently been reclassified species from some of the $C$. diphtheriae biovar Belfanti strains (Dazas et al., 2018; Badell et al., 2020). C. ulcerans, C. pseudotuberculosis and C. silvaticum infect mainly wild and domesticated mammals but can also be zoonotic (Bernard \& Funke, 2015; Dangel et al., 2020).

The $C$. diphtheria complex have an impact on public health, and also on the production of animal-based foods. Some of the species contain both DT and strains that lack the toxin. DTproducing $C$. diphtheriae strains cause cutaneous and respiratory diphtheria (Zasada, 2013; Grosse-Kock et al., 2017). The report of multidrug-resistant strains from Brazil is a new concern (Zasada, 2014; Hennart et al., 2020). DT-producing C. pseudotuberculosis from biovar equi causes Oedematous Skin Disease in buffalos (Selim et al., 2015a). C. ulcerans infects a broad range of mammal species and DT-producing strains have caused diphtheria (Hacker et al., 2016). Some non-DT producing strains of $C$. diphtheriae cause endocarditis, septic arthritis, osteomyelitis and sepsis in humans (Zasada, 2013; Grosse-Kock et al., 2017). Non-DT producing strains of C. ulcerans are associated with ulcers in humans (Hacker et al., 2016). C. pseudotuberculosis also contains non-DT strains, with those in biovar equi causing ulcerative lymphangitis in horses, and those in the biovar ovis causing caseous lymphadenitis in goat and sheep, and lymphadenitis and abscesses in humans (Selim et al., 2015b).

There are also $C$. diphtheria complex species that never produce DT but do cause disease. C. belfantii causes laryngitis and bronchopathy (Dazas et al., 2018). C. rouxii causes chronic arteritis leading to ulcerations on feet and legs, and peritonitis (Badell et al., 2020). C. silvaticum has only been isolated from pigs and roe deer to date, causing caseous lymphadenitis (Dangel et al., 2020), and is cytotoxic for human epithelial cells (Möller et al., 2021).

The host ranges and virulence mechanisms of these species are not entirely known, and better understanding of their biology could be helpful in controlling this group of pathogens. Diphtheria outbreaks were reported globally between 1921 and 2018. The disease is still endemic in some countries, with thousands of annual cases reported in Asia and Africa. The disease can emerge when the recommended vaccination programs are not applied or sustained (Sharma et al., 2019). The current vaccine is based on the DT toxoid (Rappuoli \& Malito, 2014) but does not prevent the colonization, transmission, and disease manifestation. In addition, the acquired 
84 immunity has been found to decrease with time (Truelove et al., 2020). Isolation of symptomatic 85 individuals, antitoxin and antibiotics are still essential in the control of these diseases (Truelove et 86 al., 2020). Furthermore, the diversity of DT toxin sequences across strains could reduce the 87 effectiveness of diphtheria toxoid-based vaccines and diphtheria antitoxins (Otsuji et al., 2019).

88 Another factor to consider in the control of these pathogens is that non-DT producing strains can

89

90 cause other diseases, such as ulcers and caseous lymphadenitis, the latter associated with the Phospholipase D toxin produced by C. ulcerans, C. pseudotuberculosis and C. silvaticum (Bernard \& Funke, 2015; Dangel et al., 2020).

Adaptive mutations for a specific ecological niche can be identified using genomic analyses, including genome-scale positive selection analysis (Kopac et al., 2014). At an ecological level, routine selection favors the maintenance of a stable population structure over time, while episodic selection is the effect of a sudden environmental disturbance, such as host change (Brasier, 1995). At the molecular level, positive selection can help fix adaptive mutations (Anisimova \& Liberles, 2012a). Episodes of positive selection can act on specific codons at specific times (phylogenetic branches), for which branch-site statistical models were developed (Zhang, 2005). Information on the amino acids under selection could be used for drug design (Farhat et al., 2013), or even reverse vaccinology if the amino acids are surface exposed (Goodswen, Kennedy \& Ellis, 2018).

In this work, we used a genome scale positive selection analysis to identify the genes that could be involved in ecological adaptation and identified genes that can be used to develop preventive or therapeutic strategies against this group of important pathogens.

\section{Material and Methods}

\section{Samples and taxonomy}

Episodic positive selection across species or other phylogenetic groups of the diphtheriae group was investigated using a branch-site test (Zhang, 2005). This test is more appropriate for inter-specific samples, because it assumes that the observed mutations have already been fixed by selection (Kryazhimskiy \& Plotkin, 2008; Anisimova \& Liberles, 2012a; Kosiol \& Anisimova, 2012), and one genome could represent a species. For this reason, we limited the samples to one per species, subspecies or biovar. For the foregrounds (target groups), we selected the six type strains from the $C$. diphtheria complex and other strains to represent biovars and lineages. $C$. diphtheriae biovars could not be used as foregrounds as they are united in a single clade (Sangal et al., 2014). As background, we selected 40 total genomes that included 30 representative (O'Leary et al., 2016), 8 complete and three WGS genomes of Corynebacterium species, all of which were available in the Pathosystems Resource Integration Center (PATRIC) (Davis et al., 2020). These were annotated by RASTtk (Brettin et al., 2015) and downloaded from PATRIC (Table S1).

The taxonomy of the samples was verified using TYGS (Meier-Kolthoff \& Göker, 2019). TYGS determines the closest related type strains using the MASH algorithm (Ondov et al., 2016) for entire genomes and BLASTn for 16S sequences. It calculates the pairwise distances of 16S and genome sequences using GBDP (Meier-Kolthoff et al., 2013), followed by inference of 16S and genome phylogenies based on the pairwise GBDP distances using FastME (Lefort, Desper \& Gascuel, 2015), digital DNA-DNA hybridization (dDDH) using GGDC (Meier-Kolthoff et al., 
127 2013), and deviation of G+C content. Genomes with $>70 \%$ of dDDH and $<1 \%$ of G+C content

128 deviation are considered to be in the same species (Meier-Kolthoff \& Göker, 2019).

129

\section{Positive selection analysis}

131

132

133

134

135

136

137

138

139

140

141

142

143

144

145

146

147

148

149

150

151

152

153

154

155

156

157

158

159

160

161

162

163

164

165

166

167

168

169

170
A genome-scale positive selection analysis was performed using the PosiGene pipeline (Sahm et al., 2017) on the Corynebacterium genomes. Ten foreground genomes were tested (Table S2) representing 10 target clades or subclades. Six clades represent the species from the C. diphtheria complex (C. belfantii, C. diphtheriae, C. pseudotuberculosis, C. rouxii, C. silvaticum and $C$. ulcerans), two subclades represent $C$. pseudotuberculosis (biovars equi and ovis), and two subclades representing C. ulcerans lineages (lineage 1 and 2).

In the module “create_catalog”, homologous genes were identified using BLASTp (Camacho et al., 2009) with the best-bidirectional hit criterion (Altenhoff \& Dessimoz, 2009). In the module "alignments", orthologous genes are identified, gene trees are built, and a species tree is built from the gene trees. In this module, the anchor species is the genome that the orthologous gene sequences are aligned to, and the reference species is the genome from which the gene names are extracted. C. diphtheriae NCTC $11397^{\mathrm{T}}$ was selected as both the reference and anchor genome. In the first step, orthologous gene sequences were aligned to the anchor genome sequences using CLUSTALW (Larkin et al., 2007), with the parameters' minimum identity and minimum pairs identity set to $40 \%$. The aligned sequences were filtered by GBLOCKS for gaps and unreliable alignment columns (Jordan \& Goldman, 2012). In the next step, a phylogeny was built for each gene using the parsimony method and jackknifing implemented in DNAPARS from the PHYLIP package (Felsenstein, 2005). In the third step, a species tree was built based on the gene trees consensus, using CONSENSE from the PHYLIP package. The species tree is required to test for positive selection along specific lineages (Yang \& Nielsen, 2002). The consensus tree was visualized using FigTree v1.4.4 and rooted using C. kroppenstedtii DSM 44385 as an outgroup. This strain was identified as an outgroup based on another tree generated by the same pipeline including $M$. tuberculosis H37RV to find the correct Corynebacterium species to use as an outgroup (Table S1) in that tree. The M. tuberculosis tree was not included in the downstream analysis.

In the module "positive_selection", a likelihood ratio test compares the non-synonymous to synonymous substitution rate $\omega=d_{N} / d_{S}$ in the foreground and the background. Here, $d_{N}$ is the number of non-synonymous substitutions per non-synonymous site and $d_{s}$ is the number of synonymous substitutions per synonymous site. The episodic positive selection model assumes $\omega$ $>1$ in the foreground and $\omega=0$ or $\omega<1$ in the background, while the null model assumes $\omega=0$ or $\omega<1$ for foreground and background (Yang \& Dos Reis, 2011). We considered positive selection if $p<0.05$ for False Discovery Rate, as this correction is more suitable for genome wide experiments (Storey \& Tibshirani, 2003).

Due to an assumption of no recombination by the branch-site models we used (Yang, 2005), recombination could cause false positive results (Anisimova, Nielsen \& Yang, 2003). To avoid that artifact, the genes identified as positively selected by PosiGene were then tested for intragenic recombination using PhiPack (Bruen, Philippe \& Bryant, 2006) that calculates Pairwise Homoplasy Index method (PHI) (Bruen, Philippe \& Bryant, 2006), Neighbor Similarity Score (NSS) (Jakobsen \& Easteal, 1996), and Maximum Chi-Square (Smith, 1992). In our analysis, we considered that recombination had occurred when $q<0.05$ for PHI and at least NSS 
171 or Maximum Chi-Square (Hongo et al., 2015). Genes identified as recombinant were discarded

172 for downstream analysis.

173 To minimize the false positive results caused by misalignments, frameshifts and ortholog 174 prediction (Schneider et al., 2009; Markova-Raina \& Petrov, 2011), we visually checked the 175 alignments and checked the homology prediction by comparing the orthologs protein domains 176 using PATRIC's annotation of Local and Global Families (Davis et al., 2016) and the Conserved 177 Domain Database (CDD) (Lu et al., 2020).

\section{Gene annotation}

Gene function was predicted using annotations from PATRIC and eggNOG-mapper (Huerta-Cepas et al., 2017), and InterProScan (Jones et al., 2014) was used to examine protein domains. Subcellular localization of the proteins was assessed using SufG+ v1.2.1 (Barinov et al., 2009). Protter (Omasits et al., 2014) was used to identify the position of the positively selected amino acids in relation to the cytoplasmic, transmembrane, and surface exposed portions of the proteins.

GIPSy v1.1.2 (Soares et al., 2016) was used to identify genomic islands (GIs) in $C$. diphtheriae NCTC $11397^{\mathrm{T}}$ using C. glutamicum ATCC 1302 (NC_006958.1) as the nonpathogenic reference. The islands were predicted by genes with $\mathrm{C}+\mathrm{G}$ content and codon usage deviation, presence of transposases, presence of specific genes, non-conservation in comparison to reference genome, and flanking tRNA genes. The islands were classified according to the proportion of specific genes as pathogenicity islands, metabolic islands, resistance islands and symbiotic islands. Finally, prophages were predicted using PHASTER (Arndt et al., 2016).

\section{Results}

\section{Prediction of drug and vaccine targets}

Virulence genes and drug targets were predicted using the Pipeline Builder for Identification of Targets (PBIT) (Shende et al., 2016). PBIT predicted drug targets among the cytoplasmic proteins by a subtractive approach, identifying sequences of interest using BLASTp and specific databases in the following way. First, homologs to the human proteome, anti-targets and gut microbiota proteomes were filtering out to avoid cross-reactivity of drugs. Then the essential genes were identified using the Database of Essential Genes (Zhang, 2004) and virulence genes using the Virulence Factor Database (Chen et al., 2005). The druggability of the remaining candidates was predicted by similarity to experimentally validated druggable targets from the Therapeutic Target Database (Li et al., 2018).

Vaccine targets were predicted from the transmembrane, putative surface exposed and secreted proteins predicted using SurfG+ and Vaxign (He, Xiang \& Mobley, 2010) with prediction of human MHC Class I and II epitopes.

Of the Of the 40 genomes examined, the genome of C. casei LMG S-19264 was discarded due to its being identified as Brevibacterium linens based on the TYGS pipeline (Data S1). For this reason, only 39 Corynebacterium genomes were used for the downstream analysis. Figure 1 shows the phylogeny of the Corynebacterium genomes built by the PosiGene pipeline. 
213

214

215

216

217

218

219

220

221

222

223

224

225

226

227

228

229

230

231

232

233

234

235

236

237

238

239

240

241

242

243

244

245

246

247

248

249

250

251

252

253

254

255

256
The PosiGene analysis showed zero to nine genes under positive selection $(p<0.05$ for FDR) depending upon the 10 foregrounds that were used (Cb, Cd, Cp, Cpequi, Cpovis, Cr, Cs, Cul, Cul1, Cul2 (Tables S3-12), with 22 total genes shown to be under selection. Additional analysis of the 22 genes using PhiPack $(q<0.05$ for PHI and at least NSS or Maximum ChiSquare tests) showed recombination in two out of four genes when Cd was the foreground, and one out of nine genes when Cp was used (Table S13). Manual curation of the 19 remaining proteins did not reveal any false positives caused by misalignments and ortholog prediction by comparison of protein domains (Table S14). SurfG+ predicted 15 cytoplasmic, two membrane, one putative surface exposed and one secreted protein (Table S15). Thirty-five genomic islands and two prophages were predicted in C. diphtheriae NCTC $11397^{\mathrm{T}}$ (Tables S16 and S17).

From the 19 genes that were identified as positively selected across the species, two were identified in C. diphtheriae, ten in C. pseudotuberculosis, one in C. rouxii, and six in C. ulcerans (Table 1 and Table S15). The COG categories of those genes were shown to be involved in defense, translation, energy production, and transport and metabolism of carbohydrates, amino acids, nucleotides, and coenzymes (Fig. 2). Based on our in silico prediction, fourteen genes were found to be essential, six were virulence factors, and three were found to be in genomic islands (Table 1 and Table S15).

Thirteen of the genes were identified as potential drug targets by different analyses. Three genes were predicted based on our pipeline (Tables 1 and 2, and Table S15). The other ten genes were not tagged as potential targets by the homology or druggability filters of the pipeline, but were included due to the possibility of targeting them with other methods (see Discussion section). For vaccine targets, four genes were predicted based on our pipeline (Tables 1 and 2, and Table S15).

\section{Discussion}

We identified 19 genes under positive selection, 13 potential drug targets and four potential vaccine targets. From the 13 potential drug targets, ten were includes despite not passing the homology or druggability filters of the PBIT pipeline (Tables 1 and 2, and Table S15). The problem of having homology with the human proteome or human gut microbiota proteome can be solved by screening compounds that selectively inhibit the pathogen protein (Arya et al., 2015). The druggability prediction of the PBIT pipeline is based on sequence similarity to experimentally validated targets (Shende et al., 2016). So, the lack of predicted druggability by that pipeline can be solved by prediction of druggable pockets of a protein based on its own structure (Volkamer et al., 2012). Additionally, some of those proteins are known drug targets in other species.

\section{C. diphtheriae}

In C. diphtheriae (foreground $\mathrm{Cd}$ ), we identified two genes encoding proteins predicted to be essential and involved in translation, amino acid transport and metabolism (Table 1 and Table $\mathrm{S} 15)$. The gene $a n s B$ is in GI10 and encodes secreted L-asparaginase type II which is a highaffinity enzyme that catalyzes the conversion of L-asparagine to L-aspartate and ammonia. The E. coli, Dickeya dadantii and human homologs to this gene are used for leukemia treatment, where the consequent low L-asparagine levels in plasma leads to apoptosis of the leukemia cells (Lubkowski \& Wlodawer, 2021). This gene was suggested as a candidate vaccine target due to its 
257 classification as a secreted protein and predicted epitopes (Table 2). The second protein, SSU

258 ribosomal protein S3p ( $r p s C$ ), is a 30S ribosomal subunit that binds to the initiator Met-tRNA

259 (Burd \& Dreyfuss, 1994). Possible reasons for the selective pressure on these genes could be the

260 effects on L-aspartate uptake ( $a n s B)$ and translation efficiency $(r p s C)$. rps $C$ was suggested as a

261 drug target but has homology to a protein in the human gut microbiota proteome, requiring

262 compounds that can selectively inhibit it.

\section{C. pseudotuberculosis}

When C. pseudotuberculosis was the foreground (Cp), eight positively selected genes were identified. Five of the genes were tagged as essential, and three as virulence factors. They are involved in translation, coenzyme transport and metabolism, inorganic ion transport, defense from foreign DNA, nucleotide transport and metabolism and adhesion (Table 1 and Table S15). Among the essential genes, an ABC transporter permease protein (mntC) plays a role in the transport of $\mathrm{Mn}^{2+}$ and $\mathrm{Zn}^{2+}$ (Claverys, 2001) and was classified as a virulence factor. The Dihydropteroate synthase 2 (folP2) is nonfunctional according to PATRIC annotation but functional genes with this annotation are essential for the de novo synthesis of folate (Bertacine Dias et al., 2018). The HNH endonucleases degrade foreign DNA, and can also be involved in DNA repair, replication and recombination (Wu, Lin \& Yuan, 2020). Peptide chain release factor 1 (prfA) recognizes the stop codons UAA and UAG, promoting the end of translation (Scolnick et al., 1968). The Putative phosphoglycerate mutase (pgmB) is capable of interconverting 2- and 3phosphoglycerate in glycolysis (Rigden, 2008), although this particular gene is annotated as putative.

The other three identified genes (add, spaE and the putative oxidoreductase) are not characterized as essential, so may not be suitable drug targets. Adenosine deaminase (add) catalyzes the hydrolytic deamination of adenosine into inosine (Chang et al., 1991). spaE, from

282 the operon spaDEF, encodes the minor pilin SpaE in C. diphtheriae (Mandlik et al., 2008) and is in GI5. It's ortholog in C. pseudotuberculosis also encodes the minor pilin and was first described as $s p a B$ from the operon spaABC (Trost et al., 2010). The putative oxidoreductase is a flavoenzyme with a "FAD-binding domain, ferredoxin reductase-type" (IPR017927), but its specific reaction is unknown.

Why would these particular genes be under positive selection? One could hypothesize that there would be more efficient manganese uptake ( $m n t C)$, tissue adhesion on a new host range (spaE), improved efficiency for defense against foreign DNA (HNH endonuclease), translation (prfA), and metabolism of nucleotides (add) and carbohydrates (pgmB).

The vaccine targets ( $m n t C$ and spaE) were indicated due to either their membrane location,

293

294 their predicted epitopes, and that they might have surface exposed sites that are under positive selection. There were four drug targets (Table 2). pgmB was predicted as a target by PBIT and is this same gene is a drug target in helminth parasites (Timson, 2016). folP2 was also predicted and is a well know target of sulfa and imidazole derivatives in human pathogens such as Staphylococcus aureus, M. tuberculosis, Bacillus anthracis, Streptococcus pneumoniae, Burkholderia cenocepacia and Yersinia pestis (Bertacine Dias et al., 2018). Although it was annotated as non-functional, the evidence of positive selection in this protein suggests an unknown adaptation due to specific amino acids that could be targeted. prfA has homology to human and human gut microbiota proteome and has no predicted druggability, but it is a known 
target of the drug Apidaecin in gram negative bacteria (Matsumoto et al., 2017). The HNH endonuclease had no predicted druggability.

\section{C. pseudotuberculosis biovar equi}

When C. pseudotuberculosis biovar equi was used as the foreground (Cpequi), two genes were identified as being under positive selection, and were also characterized as essential. These two genes (mapB and tyrS) are both involved in translation (Table 1 and Table S15). Methionine aminopeptidase $(\mathrm{mapB})$ cleaves the initiator methionine from newly synthesized polypeptides (Helgren et al., 2016; Pillalamarri et al., 2021). Tyrosyl-tRNA synthetase (tyrS) attaches the amino acid tyrosine to the appropriate tRNA (Hughes et al., 2020; Othman et al., 2021). These two genes could be under positive selection as it could affect translation efficiency. Both genes were predicted as homologs to human gut microbiota and have been identified as drug targets in other studies (Table 2). tyrS was predicted as a virulence factor and an ortholog from Pseudomonas aeruginosa 
341 (Peiro et al., 2019). The second gene is annotated in PATRIC as "Similar to citrate lyase beta

342 chain, 3" (citE) and is probably one of the catalytic subunits of citrate lyase, the enzyme that

343 catalyzes the cleavage of citrate to acetate and oxaloacetate during citrate fermentation

344 (Schneider, Dimroth \& Bott, 2000). Both proteins were predicted as virulence factors by PBIT.

345 The third gene encodes a hypothetical protein (ERS451417_00635) with no predicted domain or

346 cellular localization.

347

348

349

350

351

352

353

354

355

356

357

358

359

360

361

362

363

364

365

366

367

368

369

370

371

372

373

374

375

376

377

378
The two genes with predicted function (dhaK and citE) appear to be related to metabolism inside the host. In Listeria monocytogenes, Phosphoenolpyruvate-dihydroxyacetone phosphotransferase (DhaK and other subunits) is required to utilize carbon sources for amino acid synthesis inside murine macrophages (Eylert et al., 2008). In Enterococcus faecalis, mutants of citrate fermentation genes (citE and others) were less pathogenic for the model Galleria mellonella (Martino et al., 2018). These same two genes are possible drug targets (Table 2). citE was predicted as a virulence factor and drug target candidate, while dhaK was predicted as a virulence factor and suggested despite the homology to a protein in the gut microbiota homology.

\section{C. ulcerans lineage 2}

Two essential genes (Table 1 and Table S15) were identified when the $C$. ulcerans lineage 2 was used as the foreground (Cul2). The DNA polymerase III epsilon subunit ( $(n a Q)$ has a domain with 3'-5' exonuclease proofreading activity (Raia, Delarue \& Sauguet, 2019). The other gene, $c o b K$, encodes Precorrin-6A reductase which is involved in part I of the cobalamin cofactor (vitamin B12) biosynthesis pathway (Kipkorir et al., 2021). The mutations seen in these genes could provide the organism with a more efficient means of DNA replication (dnaQ) and biosynthesis of the essential cofactor cobalamin (cobK). Neither of these genes had any predictable druggability.

\section{Probable adaptations across groups}

It is reported that most of the genes identified as being under positive selection are exposed on the surface and are involved in host colonization, and resistance to phage and antibiotics (Petersen et al., 2007; Anisimova \& Liberles, 2012b). Those under positive selection that are not surface exposed have been shown to be involved in metabolism (Petersen et al., 2007; Rao, Sivakumar \& Jayakumar, 2019) or gene regulation (Zhang et al., 2011). Considering the function of the identified genes, most of the probable adaptations appear to be related to metabolism. A notable exception is in C. pseudotuberculosis, where the pilin SpaE could have enhanced adhesion to different host species tissues. Although the specific adaptations are not clear, an amino acid fixed by positive selection is an attractive target for a therapeutic molecule, as a nonsynonymous mutation that could avoid interaction would decrease fitness. These genes could be used for reverse vaccinology and in silico drug targeting methods. 


\section{Conclusion}

380 In this analysis, we predicted 19 genes with non-synonymous mutations that are probably

381 involved in adaptations found in the pathogens C. diphtheriae, C. pseudotuberculosis, C. rouxii

382 and C. ulcerans. Based on our pipeline and literature data, 13 genes are candidate drug targets

383 and four are potential vaccine targets, but their effectiveness would require experimental

384 validation.

385

\section{References}

387ltenhoff AM, Dessimoz C. 2009. Phylogenetic and Functional Assessment of Orthologs Inference 388 Projects and Methods. PLoS Computational Biology 5:e1000262. DOI: 389 10.1371/journal.pcbi.1000262.

390nisimova M, Liberles DA. 2012a. Detecting and understanding natural selection. In: Cannarozzi 391 GM, Schneider A eds. Codon Evolution: Mechanisms and Models. Oxford: Oxford University 392 Press, 73-96. DOI: 10.13140/2.1.2079.2322.

39ßnisimova M, Liberles DA. 2012b. Detecting and understanding natural selection. In: Cannarozzi 394 GM, Schneider A eds. Codon Evolution: Mechanisms and Models. Oxford: Oxford University 395 Press, 73-96. DOI: 10.13140/2.1.2079.2322.

396nisimova M, Nielsen R, Yang Z. 2003. Effect of recombination on the accuracy of the likelihood 397 method for detecting positive selection at amino acid sites. Genetics 164:1229-1236. DOI: 398 10.1093/bioinformatics/btn086.

399rndt D, Grant JR, Marcu A, Sajed T, Pon A, Liang Y, Wishart DS. 2016. PHASTER: a better, faster 400 version of the PHAST phage search tool. Nucleic acids research 44:W16-W21. DOI: 401 10.1093/nar/gkw387.

40\&rya T, Reddi R, Kishor C, Ganji RJ, Bhukya S, Gumpena R, McGowan S, Drag M, Addlagatta A. 403 2015. Identification of the Molecular Basis of Inhibitor Selectivity between the Human and 404 Streptococcal Type I Methionine Aminopeptidases. Journal of Medicinal Chemistry 58. DOI: 405 10.1021/jm501790e.

4ð6adell E, Hennart M, Rodrigues C, Passet V, Dazas M, Panunzi L, Bouchez V, Carmi-Leroy A, 407 Toubiana J, Brisse S. 2020. Corynebacterium rouxii sp. nov., a novel member of the diphtheriae 408 species complex. Research in Microbiology 171:122-127. DOI: 10.1016/j.resmic.2020.02.003. 
4\%3arinov A, Loux V, Hammani A, Nicolas P, Langella P, Ehrlichh D, Maguin E, van Guchte M de. 410 2009. Prediction of surface exposed proteins in Streptococcus pyogenes, with a potential 411 application to other Gram-positive bacteria. Proteomics 9:61-73. DOI: 10.1002/pmic.200800195. 4 Batool N, Ko KS, Chaurasia AK, Kim KK. 2020. Functional Identification of Serine 413 Hydroxymethyltransferase as a Key Gene Involved in Lysostaphin Resistance and Virulence 414 Potential of Staphylococcus aureus Strains. International Journal of Molecular Sciences 21:9135. 415 DOI: 10.3390/ijms21239135.

4Pernard AL, Funke G. 2015. Corynebacterium. In: Bergey’s Manual of Systematic of Archaea and 417 Bacteria (Online). London: John Wiley \& Sons, Bergey’s Manual Trust, 1-70. DOI: $418 \quad 10.1002 / 9781118960608$.

4Bertacine Dias M V, Santos JC, Libreros-Zúñiga GA, Ribeiro JA, Chavez-Pacheco SM. 2018. Folate 420 biosynthesis pathway: mechanisms and insights into drug design for infectious diseases. Future 421 Medicinal Chemistry 10:935-959. DOI: 10.4155/fmc-2017-0168.

473rasier CM. 1995. Episodic selection as a force in fungal microevolution, with special reference to 423 clonal speciation and hybrid introgression. Canadian Journal of Botany 73:1213-1221. DOI: 424 10.1139/b95-381.

4Æßrettin T, Davis JJ, Disz T, Edwards R a, Gerdes S, Olsen GJ, Olson R, Overbeek R, Parrello B, 426 Pusch GD, Shukla M, Thomason J a, Stevens R, Vonstein V, Wattam AR, Xia F. 2015. RASTtk: 427 A modular and extensible implementation of the RAST algorithm for building custom annotation 428 pipelines and annotating batches of genomes. Scientific Reports 5:1-6. DOI: 10.1038/srep08365.

439uen TC, Philippe HH, Bryant D. 2006. A simple and robust statistical test for detecting the presence 430 of recombination. Genetics 172:2665-81. DOI: 10.1534/genetics.105.048975.

43urd CG, Dreyfuss G. 1994. Conserved structures and diversity of functions of RNA-binding 432 proteins. Science (New York, N.Y.) 265:615-21. DOI: 10.1126/science.8036511.

433amacho C, Coulouris G, Avagyan V, Ma N, Papadopoulos J, Bealer K, Madden TL. 2009. BLAST+: 434 architecture and applications. BMC Bioinformatics 10:421. DOI: 10.1186/1471-2105-10-421.

435hang ZY, Nygaard P, Chinault AC, Kellems RE. 1991. Deduced amino acid sequence of Escherichia 436 coli adenosine deaminase reveals evolutionarily conserved amino acid residues: implications for 437 catalytic function. Biochemistry 30:2273-80. DOI: 10.1021/bi00222a033. 
438hen L, Yang J, Yu J, Yao Z, Sun L, Shen Y, Jin Q. 2005. VFDB: a reference database for bacterial 439 virulence factors. Nucleic Acids Research 33:D325-8. DOI: 10.1093/nar/gki008.

440laverys JP. 2001. A new family of high-affinity ABC manganese and zinc permeases. Research in 441 microbiology 152:231-43. DOI: 10.1016/s0923-2508(01)01195-0.

442angel A, Berger A, Rau J, Eisenberg T, Kämpfer P, Margos G, Contzen M, Busse H-J, Konrad R, 443 Peters M, Sting R, Sing A. 2020. Corynebacterium silvaticum sp. nov., a unique group of NTTB 444 corynebacteria in wild boar and roe deer. International Journal of Systematic and Evolutionary 445 Microbiology. DOI: 10.1099/ijsem.0.004195.

44ðavis JJ, Gerdes S, Olsen GJ, Olson R, Pusch GD, Shukla M, Vonstein V, Wattam AR, Yoo H. 2016. 447 PATtyFams: Protein families for the microbial genomes in the PATRIC database. Frontiers in 448 Microbiology 7:1-12. DOI: 10.3389/fmicb.2016.00118.

449avis JJ, Wattam AR, Aziz RK, Brettin T, Butler RRM, Butler RRM, Chlenski P, Conrad N, 450 Dickerman A, Dietrich EM, Gabbard JL, Gerdes S, Guard A, Kenyon RW, Machi D, Mao C, 451 Murphy-Olson D, Nguyen M, Nordberg EK, Olsen GJ, Olson RD, Overbeek JC, Overbeek R, 452 Parrello B, Pusch GD, Shukla M, Thomas C, VanOeffelen M, Vonstein V, Warren AS, Xia F, 453 Xie D, Yoo H, Stevens R. 2020. The PATRIC Bioinformatics Resource Center: Expanding data 454 and analysis capabilities. Nucleic Acids Research 48:D606-D612. DOI: 10.1093/nar/gkz943.

455azas M, Badell E, Carmi-Leroy A, Criscuolo A, Brisse S. 2018. Taxonomic status of 456 Corynebacterium diphtheriae biovar Belfanti and proposal of Corynebacterium belfantii sp. nov. 457 International Journal of Systematic and Evolutionary Microbiology 68:3826-3831. DOI: 458 10.1099/ijsem.0.003069.

4F9ylert E, Schär J, Mertins S, Stoll R, Bacher A, Goebel W, Eisenreich W. 2008. Carbon metabolism of 460 Listeria monocytogenes growing inside macrophages. Molecular Microbiology 69:1008-1017. 461 DOI: 10.1111/j.1365-2958.2008.06337.x.

462arhat MR, Shapiro BJ, Kieser KJ, Sultana R, Jacobson KR, Victor TC, Warren RM, Streicher EM, 463 Calver A, Sloutsky A, Kaur D, Posey JE, Plikaytis B, Oggioni MR, Gardy JL, Johnston JC, 464 Rodrigues M, Tang PKC, Kato-Maeda M, Borowsky ML, Muddukrishna B, Kreiswirth BN, 465 Kurepina N, Galagan J, Gagneux S, Birren B, Rubin EJ, Lander ES, Sabeti PC, Murray M. 2013. 466 Genomic analysis identifies targets of convergent positive selection in drug-resistant 467 Mycobacterium tuberculosis. Nature genetics 45:1183-9. DOI: 10.1038/ng.2747. 
468lsenstein J. 2005. PHYLIP (Phylogeny Inference Package) version 3.6.

469oodswen SJ, Kennedy PJ, Ellis JT. 2018. A Gene-Based Positive Selection Detection Approach to 470 Identify Vaccine Candidates Using Toxoplasma gondii as a Test Case Protozoan Pathogen. 471 Frontiers in Genetics 9:1-16. DOI: 10.3389/fgene.2018.00332.

4T2rosse-Kock S, Kolodkina V, Schwalbe EC, Blom J, Burkovski A, Hoskisson PA, Brisse S, Smith D, 473 Sutcliffe IC, Titov L, Sangal V. 2017. Genomic analysis of endemic clones of toxigenic and non474 toxigenic Corynebacterium diphtheriae in Belarus during and after the major epidemic in 1990s. 475 BMC Genomics 18:1-10. DOI: 10.1186/s12864-017-4276-3.

47бе Y, Xiang Z, Mobley HLT. 2010. Vaxign: The first web-based vaccine design program for reverse 477 vaccinology and applications for vaccine development. Journal of Biomedicine and 478 Biotechnology 2010. DOI: 10.1155/2010/297505.

479elgren TR, Wangtrakuldee P, Staker BL, Hagen TJ. 2016. Advances in Bacterial Methionine 480 Aminopeptidase Inhibition. Current topics in medicinal chemistry 16:397-414. DOI: 481 10.2174/1568026615666150813145410.

4\&2ennart M, Panunzi LG, Rodrigues C, Gaday Q, Baines SL, Barros-Pinkelnig M, Carmi-Leroy A, 483 Dazas M, Wehenkel AM, Didelot X, Toubiana J, Badell E, Brisse S. 2020. Population genomics 484 and antimicrobial resistance in Corynebacterium diphtheriae. Genome Medicine 12:107. DOI: 485 10.1186/s13073-020-00805-7.

4\&6ongo JA, de Castro GM, Cintra LC, Zerlotini A, Lobo FP. 2015. POTION: an end-to-end pipeline 487 for positive Darwinian selection detection in genome-scale data through phylogenetic comparison 488 of protein-coding genes. BMC genomics 16:567. DOI: 10.1186/s12864-015-1765-0.

4\&9uerta-Cepas J, Forslund K, Coelho LP, Szklarczyk D, Jensen LJ, Von Mering C, Bork P. 2017. Fast 490 genome-wide functional annotation through orthology assignment by eggNOG-mapper. 491 Molecular Biology and Evolution 34:2115-2122. DOI: 10.1093/molbev/msx148.

492ughes CA, Gorabi V, Escamilla Y, Dean FB, Bullard JM. 2020. Two Forms of Tyrosyl-tRNA 493 Synthetase from Pseudomonas aeruginosa: Characterization and Discovery of Inhibitory 494 Compounds. SLAS DISCOVERY: Advancing the Science of Drug Discovery 25:1072-1086. DOI: $49510.1177 / 2472555220934793$. 
49akobsen IB, Easteal S. 1996. A program for calculating and displaying compatibility matrices as an 497 aid in determining reticulate evolution in molecular sequences. Computer Applications in the 498 Biosciences 12:291-295. DOI: 10.1093/bioinformatics/12.4.291.

498nes P, Binns D, Chang H-Y, Fraser M, Li W, McAnulla C, McWilliam H, Maslen J, Mitchell A, 500 Nuka G, Pesseat S, Quinn AF, Sangrador-Vegas A, Scheremetjew M, Yong S-Y, Lopez R, 501 Hunter S. 2014. InterProScan 5: genome-scale protein function classification. Bioinformatics 502 30:1236-1240. DOI: 10.1093/bioinformatics/btu031.

503rdan G, Goldman N. 2012. The effects of alignment error and alignment filtering on the sitewise 504 detection of positive selection. Molecular Biology and Evolution 29:1125-1139. DOI: 505 10.1093/molbev/msr272.

506ipkorir T, Mashabela GT, de Wet TJ, Koch A, Wiesner L, Mizrahi V, Warner DF. 2021. De Novo 507 Cobalamin Biosynthesis, Transport, and Assimilation and Cobalamin-Mediated Regulation of 508 Methionine Biosynthesis in Mycobacterium smegmatis. Journal of Bacteriology 203. DOI: 509 10.1128/JB.00620-20.

510opac S, Wang Z, Wiedenbeck J, Sherry J, Wu M, Cohan FM. 2014. Genomic Heterogeneity and 511 Ecological Speciation within One Subspecies of Bacillus subtilis. Applied and Environmental 512 Microbiology 80:4842-4853. DOI: 10.1128/AEM.00576-14.

513osiol C, Anisimova M. 2012. Selection on the Protein-Coding Genome. In: Anisimova M ed. 514 Evolutionary Genomics. New York, Dordrecht, Heidelberg, London: Humana Press, 113-140. 515 DOI: 10.1007/978-1-61779-585-5_5.

516rátký M, Vinšová J, Novotná E, Mandíková J, Wsól V, Trejtnar F, Ulmann V, Stolaříková J, 517 Fernandes S, Bhat S, Liu JO. 2012. Salicylanilide derivatives block Mycobacterium tuberculosis 518 through inhibition of isocitrate lyase and methionine aminopeptidase. Tuberculosis 92. DOI: 519 10.1016/j.tube.2012.06.001.

520ryazhimskiy S, Plotkin JB. 2008. The population genetics of dN/dS. PLoS Genetics 4. DOI: 10.1371/ 521 journal.pgen.1000304.

52arkin MA, Blackshields G, Brown NP, Chenna R, Mcgettigan PA, McWilliam H, Valentin F, 523 Wallace IM, Wilm A, Lopez R, Thompson JD, Gibson TJ, Higgins DG. 2007. Clustal W and 524 Clustal X version 2.0. Bioinformatics 23:2947-2948. DOI: 10.1093/bioinformatics/btm404. 
525efort V, Desper R, Gascuel O. 2015. FastME 2.0: A Comprehensive, Accurate, and Fast Distance526 Based Phylogeny Inference Program: Table 1. Molecular Biology and Evolution 32. DOI: 527 10.1093/molbev/msv150.

5ðg YH, Yu CY, Li XX, Zhang P, Tang J, Yang Q, Fu T, Zhang X, Cui X, Tu G, Zhang Y, Li S, Yang

529 F, Sun Q, Qin C, Zeng X, Chen Z, Chen YZ, Zhu F. 2018. Therapeutic target database update 530 2018: enriched resource for facilitating bench-to-clinic research of targeted therapeutics. Nucleic 531 acids research 46:D1121-D1127. DOI: 10.1093/nar/gkx1076.

532ı S, Wang J, Chitsaz F, Derbyshire MK, Geer RC, Gonzales NR, Gwadz M, Hurwitz DI, Marchler 533 GH, Song JS, Thanki N, Yamashita RA, Yang M, Zhang D, Zheng C, Lanczycki CJ, Marchler534 Bauer A. 2020. CDD/SPARCLE: the conserved domain database in 2020. Nucleic Acids 535 Research 48:D265-D268. DOI: 10.1093/nar/gkz991.

536ubkowski J, Wlodawer A. 2021. Structural and biochemical properties of L asparaginase. The 537 FEBS Journal 288. DOI: 10.1111/febs.16042.

539andlik A, Swierczynski A, Das A, Ton-That H. 2008. Pili in Gram-positive bacteria: assembly, 539 involvement in colonization and biofilm development. Trends in microbiology 16:33-40. DOI: 540 10.1016/j.tim.2007.10.010.

54Markova-Raina P, Petrov D. 2011. High sensitivity to aligner and high rate of false positives in the 542 estimates of positive selection in the 12 Drosophila genomes. Genome research 21:863-74. DOI: 543 10.1101/gr.115949.110.

544artino GP, Perez CE, Magni C, Blancato VS. 2018. Implications of the expression of Enterococcus 545 faecalis citrate fermentation genes during infection. PLOS ONE 13:e0205787. DOI: 546 10.1371/journal.pone.0205787.

54Matsumoto K, Yamazaki K, Kawakami S, Miyoshi D, Ooi T, Hashimoto S, Taguchi S. 2017. In vivo 548 target exploration of apidaecin based on Acquired Resistance induced by Gene Overexpression 549 (ARGO assay). Scientific reports 7:12136. DOI: 10.1038/s41598-017-12039-6.

550eier-Kolthoff JP, Auch AF, Klenk HP, Göker M. 2013. Genome sequence-based species 551 delimitation with confidence intervals and improved distance functions. BMC Bioinformatics 14. 552 DOI: 10.1186/1471-2105-14-60. 
5517eier-Kolthoff JP, Göker M. 2019. TYGS is an automated high-throughput platform for state-of-the554 art genome-based taxonomy. Nature Communications 10:2182. DOI: 10.1038/s41467-019555 10210-3.

554öller J, Busch A, Berens C, Hotzel H, Burkovski A. 2021. Newly Isolated Animal Pathogen 557 Corynebacterium silvaticum Is Cytotoxic to Human Epithelial Cells. International Journal of 558 Molecular Sciences 22. DOI: 10.3390/ijms22073549.

559' Leary NA, Wright MW, Brister JR, Ciufo S, Haddad D, McVeigh R, Rajput B, Robbertse B, Smith560 White B, Ako-Adjei D, Astashyn A, Badretdin A, Bao Y, Blinkova O, Brover V, Chetvernin V, 561 Choi J, Cox E, Ermolaeva O, Farrell CM, Goldfarb T, Gupta T, Haft D, Hatcher E, Hlavina W, 562 Joardar VS, Kodali VK, Li W, Maglott D, Masterson P, McGarvey KM, Murphy MR, O’Neill K, 563 Pujar S, Rangwala SH, Rausch D, Riddick LD, Schoch C, Shkeda A, Storz SS, Sun H, Thibaud564 Nissen F, Tolstoy I, Tully RE, Vatsan AR, Wallin C, Webb D, Wu W, Landrum MJ, Kimchi A, 565 Tatusova T, DiCuccio M, Kitts P, Murphy TD, Pruitt KD. 2016. Reference sequence (RefSeq) 566 database at NCBI: current status, taxonomic expansion, and functional annotation. Nucleic Acids 567 Research 44. DOI: 10.1093/nar/gkv1189.

568masits U, Ahrens CH, Müller S, Wollscheid B. 2014. Protter: Interactive protein feature 569 visualization and integration with experimental proteomic data. Bioinformatics 30:884-886. DOI: 570 10.1093/bioinformatics/btt607.

5XZndov BD, Treangen TJ, Melsted P, Mallonee AB, Bergman NH, Koren S, Phillippy AM. 2016. 572 Mash: fast genome and metagenome distance estimation using MinHash. Genome Biology 573 17:132. DOI: 10.1186/s13059-016-0997-X.

574thman IMM, Gad-Elkareem MAM, Hassane Anouar E, Aouadi K, Snoussi M, Kadri A. 2021. New 575 substituted pyrazolones and dipyrazolotriazines as promising tyrosyl-tRNA synthetase and 576 peroxiredoxin-5 inhibitors: Design, synthesis, molecular docking and structure-activity 577 relationship (SAR) analysis. Bioorganic Chemistry 109:104704. DOI: 578 10.1016/j.bioorg.2021.104704.

5TOtsuji K, Fukuda K, Ogawa M, Saito M. 2019. Mutation and diversity of diphtheria toxin in 580 Corynebacterium ulcerans. Emerging Infectious Diseases 25:2122-2123. DOI: 581 10.3201/eid2511.181455. 
582iro C, Millard P, de Simone A, Cahoreau E, Peyriga L, Enjalbert B, Heux S. 2019. Chemical and 583 metabolic controls on dihydroxyacetone metabolism lead to suboptimal growth of Escherichia 584 coli. Applied and Environmental Microbiology 85:1-17. DOI: 10.1128/AEM.00768-19.

585etersen L, Bollback JP, Dimmic M, Hubisz M, Nielsen R. 2007. Genes under positive selection in 586 Escherichia coli. Genome Research 17:1336-1343. DOI: 10.1101/gr.6254707.

5\&्ञllalamarri V, Reddy CG, Bala SC, Jangam A, Kutty VV, Addlagatta A. 2021. Methionine 588 aminopeptidases with short sequence inserts within the catalytic domain are differentially 589 inhibited: Structural and biochemical studies of three proteins from Vibrio spp. European journal 590 of medicinal chemistry 209:112883. DOI: 10.1016/j.ejmech.2020.112883.

5qlaia P, Delarue M, Sauguet L. 2019. An updated structural classification of replicative DNA 592 polymerases. Biochemical Society Transactions 47:239-249. DOI: 10.1042/BST20180579.

5\$3ao RT, Sivakumar N, Jayakumar K. 2019. Analyses of Livestock-Associated Staphylococcus aureus 594 Pan-Genomes Suggest Virulence Is Not Primary Interest in Evolution of Its Genome. OMICS: A 595 Journal of Integrative Biology 23:224-236. DOI: 10.1089/omi.2019.0005.

5qappuoli R, Malito E. 2014. History of Diphtheria Vaccine Development. In: Burkovski A ed. 597 Corynebacterium diphtheriae and Related Toxigenic Species. Dordrecht: Springer Netherlands, 598 225-238. DOI: 10.1007/978-94-007-7624-1_11.

599igden DJ. 2008. The histidine phosphatase superfamily: structure and function. The Biochemical 600 journal 409:333-48. DOI: 10.1042/BJ20071097.

60Şhhm A, Bens M, Platzer M, Szafranski K. 2017. PosiGene: automated and easy-to-use pipeline for 602 genome-wide detection of positively selected genes. Nucleic Acids Research 45:1-11. DOI: 603 10.1093/nar/gkx179.

60\$ngal V, Burkovski A, Hunt AC, Edwards B, Blom J, Hoskisson PA. 2014. A lack of genetic basis 605 for biovar differentiation in clinically important Corynebacterium diphtheriae from whole 606 genome sequencing. Infection, Genetics and Evolution 21. DOI: 10.1016/j.meegid.2013.10.019.

6Cschneider K, Dimroth P, Bott M. 2000. Biosynthesis of the prosthetic group of citrate lyase. 608 Biochemistry 39:9438-50. DOI: 10.1021/bi000401r. 
6osehneider A, Souvorov A, Sabath N, Landan G, Gonnet GH, Graur D. 2009. Estimates of Positive 610 Darwinian Selection Are Inflated by Errors in Sequencing, Annotation, and Alignment. Genome 611 Biology and Evolution 1:114-118. DOI: 10.1093/gbe/evp012.

615colnick E, Tompkins R, Caskey T, Nirenberg M. 1968. Release factors differing in specificity for 613 terminator codons. Proceedings of the National Academy of Sciences of the United States of 614 America 61:768-74. DOI: 10.1073/pnas.61.2.768.

615elim SA, Mohamed FH, Hessain AM, Moussa IM. 2015a. Immunological characterization of 616 diphtheria toxin recovered from Corynebacterium pseudotuberculosis. Saudi Journal of 617 Biological Sciences:0-5. DOI: 10.1016/j.sjbs.2015.11.004.

6Flim SA, Mohamed FH, Hessain AM, Moussa IM. 2015b. Immunological characterization of 619 diphtheria toxin recovered from Corynebacterium pseudotuberculosis. Saudi Journal of 620 Biological Sciences:0-5. DOI: 10.1016/j.sjbs.2015.11.004.

6sharma NC, Efstratiou A, Mokrousov I, Mutreja A, Das B, Ramamurthy T. 2019. Diphtheria. Nature 622 Reviews Disease Primers 5. DOI: 10.1038/s41572-019-0131-y.

63æende G, Haldankar H, Barai RS, Bharmal MH, Shetty V, Idicula-Thomas S. 2016. PBIT: pipeline 624 builder for identification of drug targets for infectious diseases. Bioinformatics:btw760. DOI: 625 10.1093/bioinformatics/btw760.

628mith JM. 1992. Analyzing the mosaic structure of genes. Journal of Molecular Evolution 34:126627 129. DOI: 10.1007/BF00182389.

6280ares SC, Geyik H, Ramos RTJ, de Sá PHCG, Barbosa EGV, Baumbach J, Figueiredo HCP, 629 Miyoshi A, Tauch A, Silva A, Azevedo V. 2016. GIPSy: Genomic island prediction software. 630 Journal of Biotechnology 232:2-11. DOI: 10.1016/j.jbiotec.2015.09.008.

6\$10rey JD, Tibshirani R. 2003. Statistical significance for genomewide studies. Proceedings of the 632 National Academy of Sciences of the United States of America 100:9440-5. DOI: 633 10.1073/pnas.1530509100.

63ß4mson DJ. 2016. Metabolic Enzymes of Helminth Parasites: Potential as Drug Targets. Current 635 Protein \& Peptide Science 17:280-295. DOI: 10.2174/1389203717999160226180733.

6316rost E, Ott L, Schneider J, Schröder J, Jaenicke S, Goesmann A, Husemann P, Stoye J, Dorella FA, 637 Rocha FS, Soares SDC, D’Afonseca V, Miyoshi A, Ruiz J, Silva A, Azevedo V, Burkovski A, 
638 Guiso N, Join-Lambert OF, Kayal S, Tauch A. 2010. The complete genome sequence of 639 Corynebacterium pseudotuberculosis FRC41 isolated from a 12-year-old girl with necrotizing 640 lymphadenitis reveals insights into gene-regulatory networks contributing to virulence. $B M C$ 641 genomics 11:728. DOI: 10.1186/1471-2164-11-728.

6412uelove SA, Keegan LT, Moss WJ, Chaisson LH, Macher E, Azman AS, Lessler J. 2020. Clinical 643 and epidemiological aspects of diphtheria: A systematic review and pooled analysis. Clinical 644 Infectious Diseases 71:89-97. DOI: 10.1093/cid/ciz808.

645olkamer A, Kuhn D, Rippmann F, Rarey M. 2012. DoGSiteScorer: a web server for automatic 646 binding site prediction, analysis and druggability assessment. Bioinformatics 28:2074-2075. 647 DOI: 10.1093/bioinformatics/bts310.

$648 \mathrm{u}$ C-C, Lin JLJ, Yuan HS. 2020. Structures, Mechanisms, and Functions of His-Me Finger 649 Nucleases. Trends in Biochemical Sciences 45:935-946. DOI: 10.1016/j.tibs.2020.07.002.

65Yang Z. 2005. Bayes Empirical Bayes Inference of Amino Acid Sites Under Positive Selection. 651 Molecular Biology and Evolution 22:1107-1118. DOI: 10.1093/molbev/msi097.

652ang Z, Nielsen R. 2002. Codon-Substitution Models for Detecting Molecular Adaptation at 653 Individual Sites Along Specific Lineages. Molecular Biology and Evolution 19:908-917. DOI: 654 10.1093/oxfordjournals.molbev.a004148.

656ang Z, Dos Reis M. 2011. Statistical properties of the branch-site test of positive selection. 656 Molecular Biology and Evolution 28:1217-1228. DOI: 10.1093/molbev/msq303.

657asada AA. 2013. Nontoxigenic highly pathogenic clone of Corynebacterium diphtheriae, Poland, 658 2004-2012. Emerging Infectious Diseases 19:1870-1872. DOI: 10.3201/eid1911.130297.

659asada AA. 2014. Antimicrobial Susceptibility and Treatment. In: Corynebacterium diphtheriae and 660 Related Toxigenic Species. Dordrecht: Springer Netherlands, 239-246. DOI: 10.1007/978-94661 007-7624-1_12.

667hang R. 2004. DEG: a database of essential genes. Nucleic Acids Research 32:271D - 272. DOI: 663 10.1093/nar/gkh024.

664hang J. 2005. Evaluation of an Improved Branch-Site Likelihood Method for Detecting Positive 665 Selection at the Molecular Level. Molecular Biology and Evolution 22:2472-2479. DOI: 666 10.1093/molbev/msi237. 
667hang Y, Zhang H, Zhou T, Zhong Y, Jin Q. 2011. Genes under positive selection in Mycobacterium 668 tuberculosis. Computational Biology and Chemistry 35:319-322. DOI: 669 10.1016/j.compbiolchem.2011.08.001.

670 


\section{Table $\mathbf{1}$ (on next page)}

Characterization and possible application of 19 genes under positive selection in different species of the Corynebacterium diphtheria complex.

Columns: VF - virulence factor, COG - Clusters of Orthologous Groups, PS sites - positively selected sites, SE - surface exposed (sites). Column Local: C - cytoplasm, M - membrane, SE - surface exposed, S - secreted. Column COG: C - energy production and conversion, E amino acid transport and metabolism, F - nucleotide transport and metabolism, G carbohydrate transport and metabolism, $\mathrm{H}$ - coenzyme transport and metabolism, J translation, ribosomal structure and biogenesis, $\mathrm{S}$ - function unknown, $\mathrm{V}$ - defense mechanisms. Column Target: Dr - drug target, Va - vaccine target, ${ }^{1}$ - predicted by PBIT pipeline, ${ }^{2}$ - predicted by essentiality, local and Vaxign, ${ }^{3}$ - described in literature for other species, ${ }^{4}$ - suggested despite not attending one or more pipeline filters. 


\begin{tabular}{|c|c|c|c|c|c|c|c|c|c|}
\hline$n$ & Product (Gene) & PS sites & PS positions & Local & COG & Essential & $\mathbf{V F}$ & Target & GenBank ID \\
\hline & C. diphtheriae & & & & & & & & \\
\hline 1 & $\begin{array}{l}\text { L-asparaginase, type II (EC 3.5.1.1) } \\
(\text { ans } B)\end{array}$ & 3 & $69,182,339$ & $\mathrm{~S}$ & EJ & Yes & No & $\mathrm{Va}^{2}$ & ERS451417_00414 \\
\hline \multirow[t]{2}{*}{2} & $\begin{array}{l}\text { SSU ribosomal protein } \mathrm{S} 3 \mathrm{p}(\mathrm{S} 3 \mathrm{e}) \\
(\operatorname{rps} C)\end{array}$ & 1 & 89 & $\mathrm{C}$ & $\mathrm{J}$ & Yes & No & $\mathrm{Dr}^{4}$ & ERS451417_00402 \\
\hline & C. pseudotuberculosis & & & & & & & & \\
\hline 3 & $\begin{array}{l}\text { ABC transporter, permease protein } \\
(m n t C)\end{array}$ & 1 & 111 & M & $\mathrm{P}$ & Yes & Yes & $\mathrm{Va}^{2}$ & ERS451417_00548 \\
\hline 4 & Adenosine deaminase $(a d d)$ & 1 & 25 & $\mathrm{C}$ & $\mathrm{F}$ & - & No & - & ERS451417_00570 \\
\hline 5 & Adhesin SpaE (spaE) & 20 & $\begin{array}{l}23,33,35, \\
108,119, \\
122,125, \\
223,232, \\
238,239, \\
243,244, \\
247,251, \\
252,253, \\
255,257,259\end{array}$ & SE & - & No & Yes & $\mathrm{Va}^{2}$ & ERS451417_00159 \\
\hline 6 & $\begin{array}{l}\text { Dihydropteroate synthase } 2 \\
\text { (nonfunctional) }(f o l P)\end{array}$ & 2 & 135,158 & $\mathrm{C}$ & $\mathrm{H}$ & Yes & No & $\operatorname{Dr}^{1,3}$ & ERS451417_00887 \\
\hline 7 & $\mathrm{HNH}$ endonuclease & 4 & $48,111,284$ & $\mathrm{C}$ & $\mathrm{V}$ & Yes & No & $\mathrm{Dr}^{4}$ & ERS451417_00880 \\
\hline
\end{tabular}


352

8 Peptide chain release factor $1(\operatorname{prf} A)$

9 Putative oxidoreductase

10 Putative phosphoglycerate mutase $(\operatorname{pgmB})$

\section{C. pseudotuberculosis equi}

11 Methionine aminopeptidase (EC 3.4.11.18) (mapB)

12 Tyrosyl-tRNA synthetase (EC 6.1.1.1) (tyr $S)$

\section{C. rouxii}

13 Hypothetical protein

\section{C. ulcerans}

14 Serine hydroxymethyltransferase (EC 2.1.2.1) (glyA)

\section{C. ulcerans lineage 1}

15 Hypothetical protein

16 Phosphoenolpyruvate-

60

$33,42,48$

C J

Yes

No

$\operatorname{Dr}^{3}$

ERS451417_00951

$52,109,209$,

226,285

269,143

C

G

Yes

No

$\operatorname{Dr}^{1},{ }^{3}$

ERS451417 02267

2

$23,58,59$

403

4

$5,74,95,154$

M

S

385

$1 \quad 32$

4248,251 ,

C $\quad$ G

$\begin{array}{ll}\mathrm{Dr}^{4} & \text { ERS451417_00635 } \\ \mathrm{Dr}^{4} & \text { ERS451417 } 02360\end{array}$ 
dihydroxyacetone

255,256

phosphotransferase,

dihydroxyacetone binding subunit

DhaK (dnaK)

17 Similar to citrate lyase beta chain, $3 \quad 1 \quad 235$

C G Yes Yes

$\operatorname{Dr}^{1}$

ERS451417_00750 (citE)

\section{C. ulcerans lineage 2}

18 DNA polymerase III epsilon subunit 1 (EC 2.7.7.7) (dnaQ)

19 Precorrin-6A reductase (EC 1.3.1.54) $\quad 1 \quad 206$

No

$\mathrm{Dr}^{4}$

ERS451417_00985 (cobK) 


\section{Table 2 (on next page)}

Final drug and vaccine target candidates for Corynebacterium species based on a positive selection analysis by foreground, application, and priority.

PS sites - positively selected sites. 


\begin{tabular}{|c|c|c|c|c|c|c|}
\hline Foreground & Application & Priority & Product (Gene) & $\begin{array}{l}\text { PS sites (exposed } \\
\text { sites) }\end{array}$ & Local & GenBank ID \\
\hline $\mathrm{Cd}$ & Drug target & $\begin{array}{l}1 \text { (gut microbiota } \\
\text { homolog) }\end{array}$ & $\begin{array}{l}\text { SSU ribosomal protein } \mathrm{S} 3 \mathrm{p}(\mathrm{S} 3 \mathrm{e}) \\
(r p s C)\end{array}$ & 89 & $\mathrm{C}$ & ERS451417_00402 \\
\hline $\mathrm{Cd}$ & Vaccine & 1 & L-asparaginase $($ ans $B)$ & $\begin{array}{l}69,182,339(69, \\
182,339)\end{array}$ & $\mathrm{S}$ & ERS451417_00414 \\
\hline $\mathrm{Cp}$ & Drug target & 1 & $\begin{array}{l}\text { Putative phosphoglycerate mutase } \\
\text { (pgmB) }\end{array}$ & 69,143 & $\mathrm{C}$ & ERS451417_02267 \\
\hline $\mathrm{Cp}$ & Drug target & $\begin{array}{l}2 \text { (predicted as } \\
\text { nonfunctional) }\end{array}$ & $\begin{array}{l}\text { Dihydropteroate synthase } 2 \\
\text { (nonfunctional) }(\text { folP2) }\end{array}$ & 135,158 & $\mathrm{C}$ & ERS451417_00887 \\
\hline $\mathrm{Cp}$ & Drug target & $\begin{array}{l}3 \text { (human and gut } \\
\text { microbiota homolog, } \\
\text { no predicted } \\
\text { druggability) }\end{array}$ & Peptide chain release factor 1 ( $p r f A)$ & 60 & $\mathrm{C}$ & ERS451417_00951 \\
\hline $\mathrm{Cp}$ & Drug target & $\begin{array}{l}4 \text { (no predicted } \\
\text { druggability) }\end{array}$ & HNH endonuclease & $48,111,284,352$ & $\mathrm{C}$ & ERS451417_00880 \\
\hline $\mathrm{Cp}$ & Vaccine & 1 & Adhesin SpaE (spaE) & $\begin{array}{l}23,33,35,108,119, \\
122,125,223,232, \\
238,239,243,244, \\
247,251,252,253, \\
255,257,259(23, \\
33,35,108,119, \\
122,125,223)\end{array}$ & SE & ERS451417_00159 \\
\hline $\mathrm{Cp}$ & Vaccine & $\begin{array}{l}2 \text { (no exposed PS } \\
\text { site) }\end{array}$ & $\begin{array}{l}\mathrm{ABC} \text { transporter, permease protein } \\
(m n t C)\end{array}$ & 111 & $\mathrm{M}$ & ERS451417_00548 \\
\hline Cpequi & Drug target & $\begin{array}{l}1 \text { (virulence factor, } \\
\text { more PS sites, gut } \\
\text { microbiota homolog, } \\
\text { target in literature) }\end{array}$ & Tyrosyl-tRNA synthetase (tyrS) & $23,58,59,403$ & $\mathrm{C}$ & ERS451417_01169 \\
\hline Cpequi & Drug target & $\begin{array}{l}2 \text { (less PS sites, gut } \\
\text { microbiota homolog, } \\
\text { target in literature) }\end{array}$ & Methionine aminopeptidase (mapB) & 96,97 & $\mathrm{C}$ & ERS451417_01521 \\
\hline
\end{tabular}




\begin{tabular}{|c|c|c|c|c|c|c|}
\hline $\mathrm{Cr}$ & Vaccine & 1 & Hypothetical protein & $5,74,95,154(154)$ & $\mathrm{M}$ & ERS451417_00470 \\
\hline $\mathrm{Cul}$ & Drug target & $\begin{array}{l}1 \text { (human and gut } \\
\text { microbiota homolog) }\end{array}$ & $\begin{array}{l}\text { Serine hydroxymethyltransferase } \\
(\text { gly } A)\end{array}$ & 385 & $\mathrm{C}$ & ERS451417_00836 \\
\hline Cul1 & Drug target & 1 (virulence factor) & $\begin{array}{l}\text { Similar to citrate lyase beta chain } \\
\text { (citE) }\end{array}$ & 235 & $\mathrm{C}$ & ERS451417_00750 \\
\hline Cull & Drug target & $\begin{array}{l}2 \text { (virulence factor, } \\
\text { gut microbiota } \\
\text { homolog) }\end{array}$ & $\begin{array}{l}\text { Phosphoenolpyruvate- } \\
\text { dihydroxyacetone } \\
\text { phosphotransferase, } \\
\text { dihydroxyacetone binding subunit } \\
\text { DhaK (dhaK) }\end{array}$ & $248,251,255,256$ & - & ERS451417_02360 \\
\hline Cull & Drug target & $\begin{array}{l}3 \text { (hypothetical } \\
\text { protein, no predicted } \\
\text { druggability) }\end{array}$ & Hypothetical protein & 32 & $\mathrm{C}$ & ERS451417_00635 \\
\hline $\mathrm{Cul} 2$ & Drug target & $\begin{array}{l}\text { Equal. No predicted } \\
\text { druggability }\end{array}$ & $\begin{array}{l}\text { DNA polymerase III epsilon } \\
\text { subunit }(\text { dnaQ })\end{array}$ & 142 & $\mathrm{C}$ & ERS451417_00985 \\
\hline $\mathrm{Cul} 2$ & Drug target & $\begin{array}{l}\text { Equal. No predicted } \\
\text { druggability }\end{array}$ & Precorrin-6A reductase $(\operatorname{cobK})$ & 206 & $\mathrm{C}$ & ERS451417_01234 \\
\hline
\end{tabular}


Figure 1

The Corynebacterium species tree that was generated by the PosiGene pipeline, using CONSENSE from the PHYLIP package.

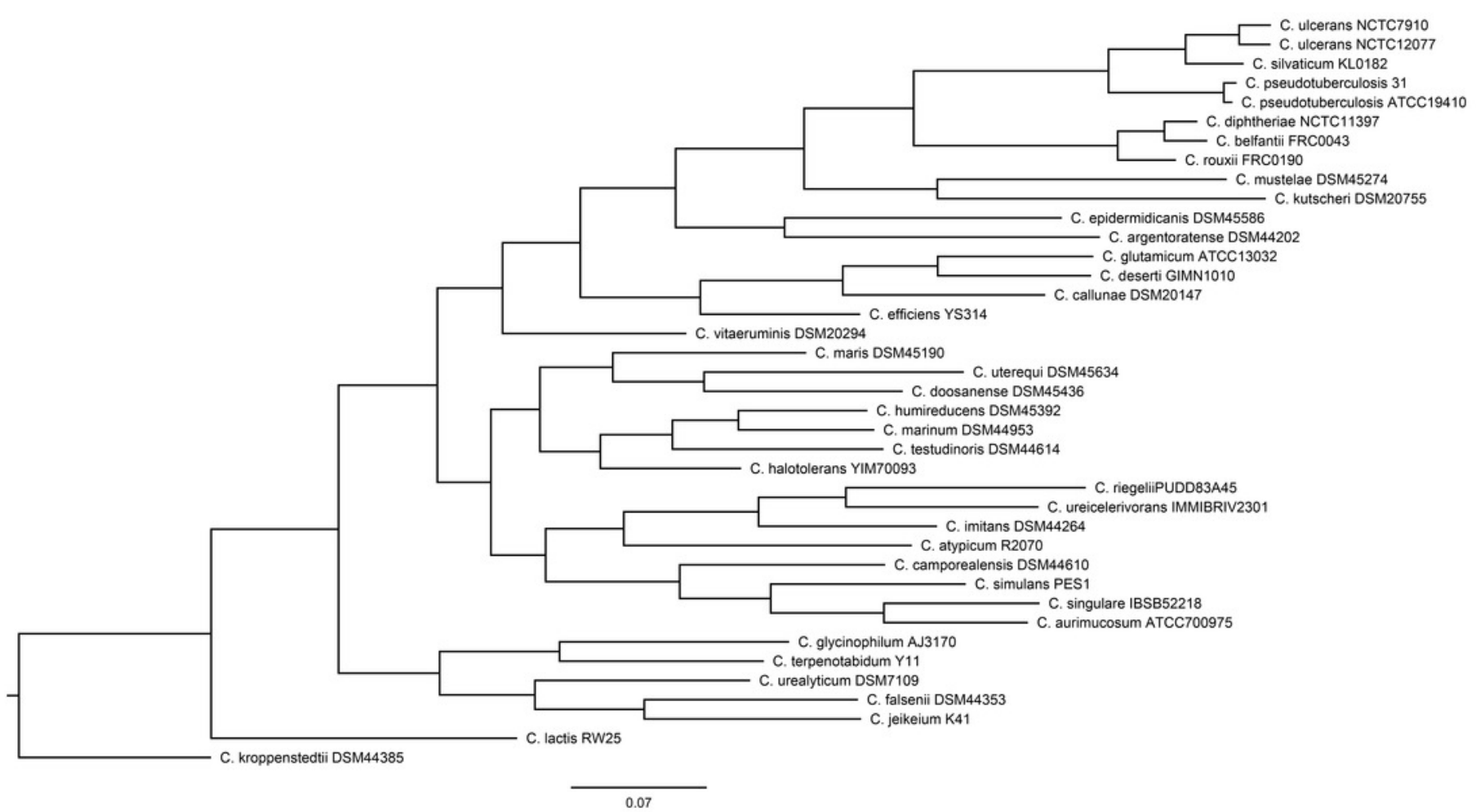


Figure 2

Distribution of 19 genes under positive selection in COG categories.

Target groups: Cd - C. diphtheriae, Cp - C. pseudotuberculosis, Cpequi - C.

pseudotuberculosis biovar equi, $\mathrm{Cr}-\mathrm{C}$. rouxii, Cul - C. ulcerans, Cul1 - C. ulcerans lineage 1 , Cul2 - C. ulcerans lineage 2. COG categories: C-Q - metabolism, J-L - information storage and processing, M-V - cellular processes and signaling, S - poorly characterized.

\section{Genes in COG categories}

2.5

2

1.5

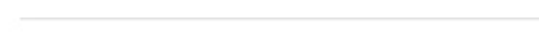

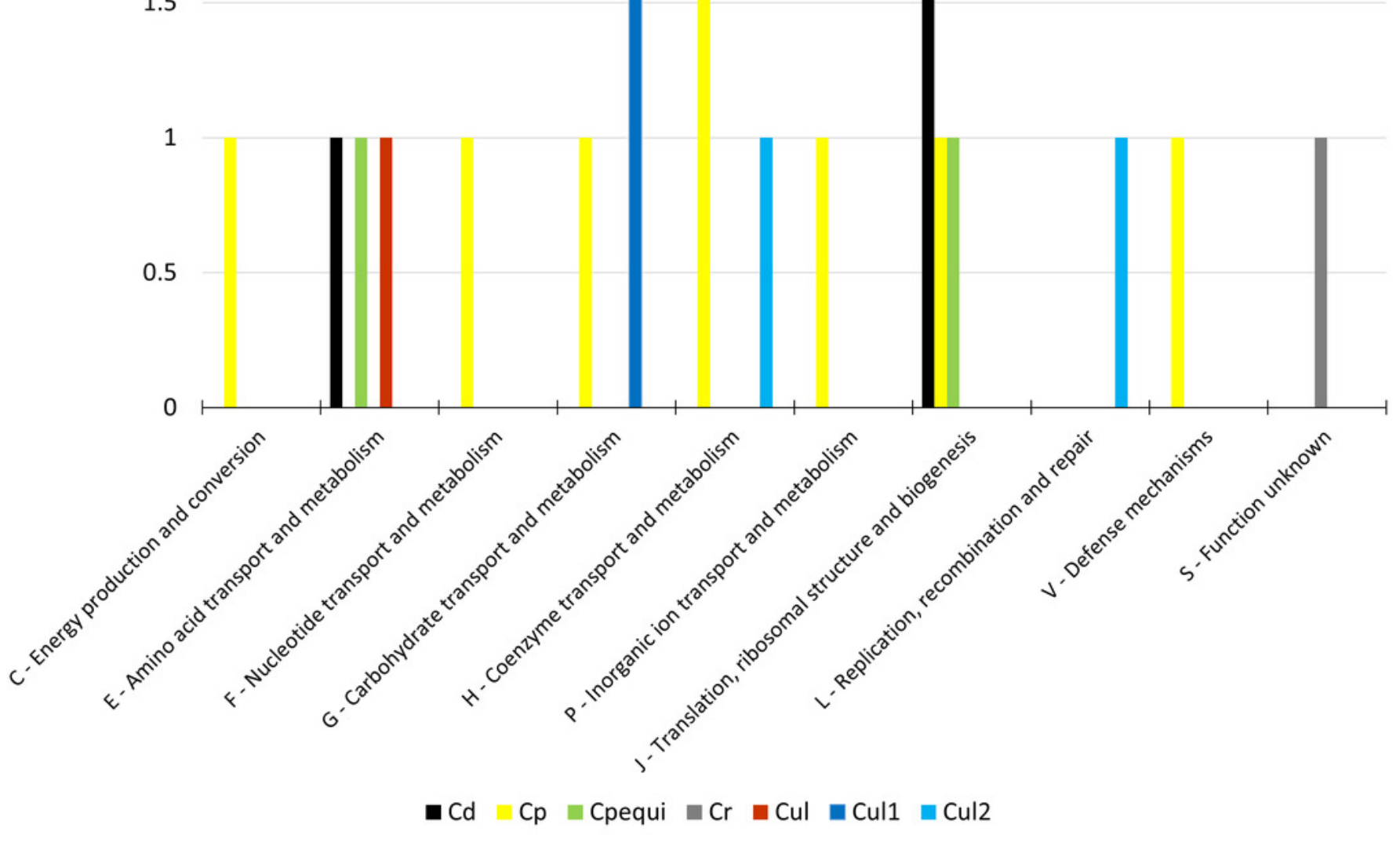

